\title{
Control of chaos and synchronization
}

\author{
Henk Nijmeijer* \\ Department of Applied Mathematics, University of Twente, P.O. Box 217, 7500 AE Enschede, Netherlands
}

\begin{abstract}
This note presents a brief introduction in the field of control of chaos and chaos synchronization. It is argued that both subjects, being very popular among physicists, also deserve to be studied from a control perspective. (C) 1997 Elsevier Science B.V.
\end{abstract}

\section{Introduction}

Perhaps one of the most fascinating and rapidly growing disciplines today is chaos. 'Chaos making a new science' is the title of a book by James Gleick, [6], and this is possibly the way many people view it nowadays. There is tremendous activity in many fields dealing with chaos, as for instance, economics, biology, astronomy, communication theory, physics, mechanics and, of course, mathematics. Research includes experimental, numerical and theoretical work, and although within mathematics chaos is a central theme in the study of dynamical systems, still many aspects are not, or not completely, understood. Given the huge interest in the subject, it comes as no surprise that chaos has also entered the control scene. In fact, chaotic dynamics and control meet in - at least two distinct points, namely synchronization and what is called control of chaos. Both items have been intensively explored in the last decade or so, see e.g. the electronic bibliography [1], or the books $[7,10]$. Remarkably enough, most of the literature from [1] is aside from the mainstream systems and control literature and it is therefore that we focus here on control of chaos and synchronization.

The aim of this paper and also of the complete special issue is to bridge a gap between, on the one hand,

\footnotetext{
*Fax: +31534340733; e-mail: h.nijmeijer@math.utwente.nl.
}

'standard' control theory and, on the other hand, chaos control. Hopefully, by presenting some of the current trends in the field of chaos control, the reader may become convinced that some useful scientific interactions between the two themes should become possible.

The organization of this note is as follows. In the next section, we briefly introduce by means of two examples what chaotic dynamics are. Then, in Sections 3 and 4 we describe what control of chaos and synchronization is about.

\section{Examples of chaotic systems}

In this section we describe two illustrative examples of chaotic dynamical systems, or more precisely, systems that possess a chaotic attractor. The examples are, respectively, chosen in discrete time and continuous time, as to illustrate that chaos occurs in both domains.

Example 2.1 (Devaney [2] and Drazin [5]). The Hénon map describes a difference equation on $\mathbb{R}^{2}$ via

$$
\left(\begin{array}{c}
x_{k+1} \\
y_{k+1}
\end{array}\right)=\left(\begin{array}{c}
y_{k}+1-1.4 x_{k}^{2} \\
0.3 x_{k}
\end{array}\right) \text {. }
$$

One may verify, by iteration of (1) from arbitrary initial conditions $\left(x_{0}, y_{0}\right) \in \mathbb{R}^{2}$, that the dynamics (1) have a strange attractor, that is an attracting set $A_{1}$ 
having a structure quite different from an equilibrium point or periodic orbit. Indeed, the dynamics (1) chaotically wanders around in $A_{1}$, and the structure of $A_{1}$ itself is fairly complex. For instance, the box-counting dimension or, sometimes called fractal dimension, is noninteger valued, and, in this case equals 1.2. Here, the dimension is defined as that number $d$ for which the set $A_{1}$ is covered by $N(\varepsilon) \sim C_{0} \varepsilon^{-d}$ squares of side $\varepsilon$, when $\varepsilon \rightarrow 0$ and where $C_{0}>0$ is a constant. It is also not difficult to see that $A_{1}$ contains countably many periodic points of arbitrary periods [3].

Example 2.2 (Drazin [5]). The Lorenz system is obtained as an extreme simplification of a model describing the weather and is described as a continuous time dynamical system in $\mathbb{R}^{3}$ :

$\dot{x}=\sigma(y-x)$,

$\dot{y}=r x-y-x z$,

$\dot{z}=x y-b z$.

For $\sigma=16, r=45.6$ and $b=4$ (as with many other choices) the dynamics (2) have again a chaotic attractor $A_{2}$. One may visualize $A_{2}$ by simulating arbitrary solutions of (2). A few facts about $A_{2} . A_{2}$ contains 'many' period orbits of (2); we will explain shortly what 'many' means here. $A_{2}$ has again a noninteger valued box-counting dimension (like was the case for $A_{1}$ ). $A_{2}$ has a further property (like $A_{1}$ has) which can be explained as follows. The dynamics (1) has sensitive dependence on initial conditions, i.e., there exists $\beta>0$ such that for any $p \in \mathbb{R}^{3}, \forall \varepsilon>0$, there is a $q \in \mathbb{R}^{3}$ with $|q-p|<\varepsilon$ such that the solutions $\varphi^{T}(p)$ and $\varphi^{T}(q)$ have a distance larger than $\beta$. Note that $\varphi^{T}(p)$ is the solution at time $T$ of the dynamical system (2) starting at $t=0$ in $p$.

The above two examples clearly demonstrate crucial aspects of a chaotic attractor. As a definition one says that a dynamical system $\Sigma$ has a chaotic attractor $A$ if [2]

(i) The set $A$ acts as an attractor for $\Sigma$ (i.e. is the closure of all positive-time limit points of $\Sigma$ ).

(ii) The periodic points of $\Sigma$ are dense in $A$.

(iii) $\Sigma$ depends sensitively on initial conditions.

Often, an extra requirement imposed is that $\Sigma$ acts transitively on $A$, that is for any pair of points $p, q \in A$ and any $\varepsilon>0$, there is a point $z \in A$ such that $|z-p|<\varepsilon$ and the orbit of $\Sigma$ through $z$ comes within distance $\varepsilon$ of $q$. Of course, a lot more can be said about chaotic attractors, see, e.g. $[2,3,5]$ but for what follows the above may suffice. However, it should be mentioned that chaos as defined above occur in very many situations; it is not a rare phenomenon 'discovered' by mathematicians but it seemingly exists in large classes of nonlinear systems. A formal proof that systems like (1) and (2) do exhibit chaos as defined here is far from trivial and is very recent. For many other systems the existence of chaos is at best only conjectured. This is (partly) the reason for the huge interest in chaotic dynamics and may also stimulate interest in the control community for such complex behavior.

Remark. It is useful to note that in many situations it is not at all clear whether a given data set or time series is the net result of a deterministic, possibly chaotic, dynamical system like (1) or (2). Or is it, on the other hand, a time series coming from a stochastic process? This sort of time-series analysis is of key importance in the study of chaotic systems and is also essential in control of chaos; see, for instance, [10] or [14].

\section{Control of chaos}

In control of chaos the basic setting can be described as follows. Suppose a dynamical system $\Sigma$ in continuous or discrete time is given and assume that $\Sigma$ possesses a chaotic attractor $A$. Thus, typical examples are given as (1) or (2). Moreover, assume that in $\Sigma$ control actions can be performed; this can be either by means of parameter changes, but more likely, by means of an extra input vector field. For instance, one could add in (2) a term like $u *(001)^{T}$. In any case $\Sigma$ itself can be viewed as the nominal (uncontrolled) system. In control of chaos literature one deals with a tracking problem, which can be stated as follows. Let $x_{d}(t), t \geqslant 0$, be a trajectory of $\Sigma$ belonging to the chaotic attractor $A$. So $x_{d}(t), t \geqslant 0$, is a trajectory of the nominal system and the corresponding input-trajectory is $u_{d}(t)=0, t \geqslant 0$. Since $A$ is chaotic, one often choses $x_{d}(t), t \geqslant 0$ as a fixed point or periodic orbit of $\Sigma$ and the problem is to design a suitable controller that guarantees that, independent of the initial condition, the closed-loop system dynamics converges to $x_{d}(t)$. It turns out that the above tracking problem, under suitable assumptions on the controlled version of $\Sigma$, admits a local feedback solution that achieves the desired tracking with small bounded controls; see, for instance, the first paper in this direction by Ott et al. [9], where what is now 
called the OGY-method is described. In the particular case where $x_{d}(t)=x_{d}$ (= constant), with $x_{d} \in A$, it is worth noting that the uncontrolled dynamics - thus the nominal chaotic system - has the property that, given the properties of a chaotic attractor, any trajectory will come, afte:: a possibly long period of time, arbitrarily close to $x_{d}$. But after the close encounter, the trajectory of $\Sigma$ will rapidly move away from $x_{d}$, and then return arbitrarily close to $x_{d}$ after some (long) period of time, and so on. Clearly, what the controller should do, is to assure that once the system is close to $x_{d}$, then it will remain there, and asymptotically converges towards $x_{d}$. In itself, $x_{d}$ or any other point of the chaotic attractor $A$, is 'approximately' reachable from any point outside $A$, without using any control; a situation uncommon in the study of nonlinear local controllability.

The ideas as proposed in [9], as well as various other controller schemes; see, for example, [7], have found very rapid application in a number of interesting directions; see, e.g. $[11,4]$. Experimental work includes lasers, chemical systems and biological systems, where, in particular, the examples of controlling a heart tissue or brain tissue are very surprising. Although lots of questions are still open in this field, and in particular, as far as the applications go, it seems fair to say that the control of chaos literature has done one thing unusual in control, namely exploiting chaos. This is what perhaps the control community could learn from the above mentioned references. On the other hand, it is fair to say that the control of chaos techniques are rather straightforward and open to further advanced control methods as being studied within the control community.

Remark. It is interesting to mention that several of the forementioned control of chaos applications do not require a complete dynamic model as in the examples (1) or (2) but only on an available time series of the system, see [10].

\section{Synchronization}

In a completely different way, chaos has been used in what is called synchronization. Synchronization of two dynamical systems has been observed in many cases. Perhaps one of the earliest accounts was given by $\mathrm{C}$. Huijgens in the 17 th century, who observed that two pendulum clocks attached to a bar, will soon move with equal or opposite phase. The first idea of chaos synchronization is perhaps due to Pecora and Carroll who observed that two copies of the chaotic Lorenz-systems (2) may synchronize if in one of the copies the $x$-component of the other copy is plugged in, cf. [12]. In other words, the state-error between the two (chaotic) systems goes asymptotically to zero, despite the unpredictable motions in each of the separate Lorenz-systems (because of the sensitive dependence on initial conditions). After the Pecora-Carroll example numerous other examples of (seemingly) synchronized motions have been reported, see also [4], although in most cases no rigorous mathematical proof for the synchronization could be given. On the other hand, synchronization can be cast in a 'standard' control context in case one is allowed to construct one of the two systems. In that case, the synchronization reduces to an observer problem, cf. $[8,13]$ and potentially much work can be done using this perspective. Indeed it is not hard to conjecture that chaos synchronization can be very fruitfully treated within a control context. Also, if both systems are given in advance, but one is able to control one of the two systems, the problem of synchronizing the two systems can be cast in a usual control frame, although no general solution is yet available. Chaos synchronization has potential applications in signal processing and secure communications, since a message signal can be 'masked' with a chaotic signal coming from a system $\Sigma$, which at the receiver's end may be decoded by the reconstructed system state, $\mathrm{cf}$. [4, 12].

\section{Concluding remarks}

Control of chaos and chaos synchronization are two subjects which are presently very popular among physicists and others. We have briefly introduced these subjects and argued that there is room for a controlengineering approach. On the other hand, from the chaos control literature one may extract some useful ideas uncommon among the control community. We hope that this special issue will provide a positive impetus to a fruitful dialogue between the control engineering and chaos researchers community.

\section{References}

[1] G. Chen, Control and synchronization of chaos, a bibliography, Department of Electrical Engineering, University of Houston, TX, USA, available via ftp: ftp.egr.uh. 
edu/pub/TeX/chaos.tex (login name and password both "anonymous"), 1997.

[2] R.L. Devaney, An Introduction to Chaotic Dynamical Systems, 2nd ed., Addison-Wesley, Redwood City, CA, 1989.

[3] R.L. Devaney, A First Course in Chaotic Dynamical Systems, Addison-Wesley, Reading, CA, 1992.

[4] W.L. Ditto, L.M. Pecora, Mastering chaos, Scientific American 129 (1993) 78-84.

[5] P.G. Drazin, Nonlinear Systems, Cambridge University Press, Cambridge, 1992.

[6] J. Gleich, Chaos, Making a New Science, Cardinal, London, 1988.

[7] T. Kapitaniak, Controlling Chaos, Academic Press, San Diego, CA, 1996.

[8] H. Nijmeijer, I. Mareels, An observer looks at synchronization, Memorandum 1324, Department of Applied Mathematics, University of Twente, 1996, IEEE Trans. Circuits and Systems I, to appear.
[9] E. Ott, C. Grebogi, J.A. Yorke, Controlling chaos, Phys. Rev. Lett. 64 (1990) 1196-1199.

[10] E. Ott, T. Sauer, J.A. Yorke (Eds.), Coping with Chaos: Analysis of Chaotic Data and the Exploitation of Chaotic Systems, Wiley, Interscience, New York, 1994.

[11] E. Ott, M. Spano, Controlling chaos, Physics Today (1995) 34-40.

[12] L. Pecora, T. Carrol, Synchronization in chaotic systems, Phys. Rev. Lett. 64 (1990) 821-824.

[13] J.H. Peng, E.J. Ding, M. Ding, W. Yang, Synchronizing hyperchaos with a scalar transmitted signal, Phys. Rev. Lett. 76 (1996) 904-907.

[14] F. Takens, Detecting strange attractors in turbulence, in: A. Dold, B. Eckmann (Eds.), Dynamical Systems and Turbulence, Warwick 1980, Lect. Notes in Mathematics, vol. 898, Springer, Berlin, 1981. 